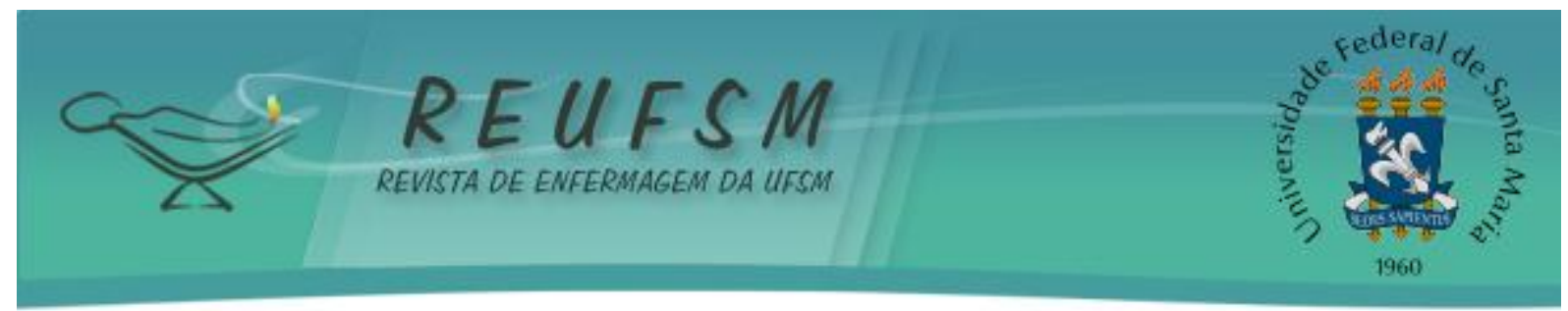

ARTIGO ORIGINAL

\title{
GRUPO DE CAMINHADA: FATOR DE PROTEÇÃO PARA EVENTOS CARDIOVASCULARES EM MULHERES HIPERTENSAS ${ }^{1}$
}

\author{
WALKING GROUP: PROTECTION FACTOR FOR CARDIOVASCULAR EVENTS IN \\ HYPERTENSIVE WOMEN
}

\section{GRUPO DE CAMINATA: FACTOR DE PROTECCIÓN PARA LOS EVENTOS CARDIOVASCULARES EN MUJERES HIPERTENSAS}

\author{
Luciano Faustino da Silva² \\ Érica Aparecida de Moraes Silva ${ }^{3}$ \\ Ricardo Castanho Moreira ${ }^{4}$ \\ Maria de Fátima Mantovani ${ }^{5}$
}

RESUMO: Objetivo: comparar a diferença de médias do Escore de Risco Cardiovascular de Framinghan (ERCF) em mulheres com hipertensão arterial que participavam de um grupo de caminhada com as que não participavam. Método: estudo transversal, realizado em uma Unidade Básica de Saúde (UBS) com Estratégia Saúde da Família (ESF), no município de Bandeirantes-PR, no período de junho a agosto de 2008, com 40 mulheres hipertensas, divididas em dois grupos: Grupo 1-Caminha e Grupo 2-Não Caminha. Os instrumentos utilizados foram formulário do perfil sociodemográfico, clínico e laboratorial; e o ERCF. Resultados: houve diferença estatisticamente significativa das médias das pontuações obtidas pelos grupos. O Grupo 1-Caminha apresentou pontuação média de 8,85 e o Grupo 2-Não Caminha 12,15, classificando-se como baixo e médio risco para evento cardiovascular, respectivamente. Conclusão: a caminhada é um importante fator de proteção para as doenças cardiovasculares.

Descritores: Mulheres; Hipertensão; Caminhada; Prevenção secundária; Isquemia miocárdica.

ABSTRACT: Objective: to compare the mean difference of Framingham's Cardiovascular Risk Score (FCRS) in women with hypertension who participated in a walking group with those who did not participate. Method: cross-sectional study held in a Community Health Unit (CHU) at the Family Health Strategy (FHS), in Bandeirantes-PR town, in the period from June to August 2008, with 40 hypertensive women, divided into two groups: Group 1Walk and Group 2-No Walk. The instruments used were form sociodemographic, clinical and laboratory, and FCRS. Results: there was statistical difference of mean scores obtained by the groups. The Group 1-Walk presented an average score of 8.85 and Group 2-No Walk 12.15, being classified as low and medium risk for cardiovascular events, respectively. Conclusion: walking is an important protective factor for cardiovascular disease.

Descriptors: Women; Hypertension; Walking; Secundary prevention; Myocardial ischemia.

\footnotetext{
${ }^{1}$ Trabalho de Conclusão de Curso, apresentado ao curso de enfermagem da Universidade Estadual do Norte do Paraná (UENP), Campus Luiz Meneghel (CLM).

${ }^{2}$ Enfermeiro. Especialista em Urgência e Emergência. Secretaria Municipal de Saúde de Arapoti-PR. E-mail: lucianojbt@hotmail.com

${ }^{3}$ Enfermeira. Graduada pela UENP/CLM. Secretaria Municipal de Saúde de São José da Boa Vista. E-mail: erica_mo@hotmail.com

${ }^{4}$ Enfermeiro. Doutorando em Enfermagem pela UFPR. Professor da UENP/CLM. E-mail: ricardocastanho@uenp.edu.br

${ }^{5}$ Enfermeira. Doutora em Enfermagem. Professora do PPGENF da Universidade Federal do Paraná. Bolsista Produtividade 2 CNPq. E-mail: mfatimamantovani@ufpr.br
} 


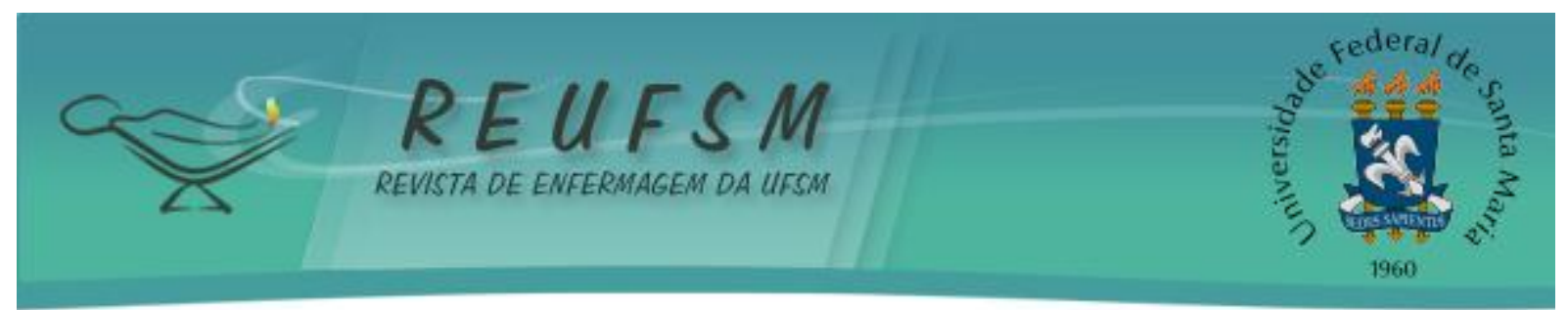

RESUMÉN: Objetivo: comparar la diferencia de la media de la Puntuación de Riesgo Cardiovascular de Framingham (PRCF) en mujeres con hipertensión que participaron en un grupo de caminantes con las que no participaron. Método: estudio transversal realizado en una Unidad Básica de Salud (UBS) con la Estrategia de Salud de la Familia (ESF), en la ciudad de Bandeirantes-PR, en el período de junio a agosto de 2008, con 40 mujeres hipertensas, divididas en dos grupos: Grupo 1-Camina y el Grupo 2-No Camina. Los instrumentos utilizados fueron el formulario sociodemográfico, clínico y de laboratorio, y PRCF. Resultados: observó una diferencia estadísticamente significativa de las puntuaciones medias obtenidas por los grupos. El Grupo 1-Camina presentó una puntuación media de 8,85 y el Grupo 2-No Camina 12,15, con clasificación de riesgo bajo y medio a eventos cardiovasculares, respectivamente. Conclusión: caminar es un importante factor protector de enfermedad cardiovascular.

Descriptores: Mujeres; Hipertensión; Caminata; Prevención secundaria; Isquemia miocárdica.

\section{INTRODUÇÃO}

As Doenças Crônicas Não Transmissíveis (DCNT) são as principais causas de morte no mundo, e corresponderam a $68 \%$ dos óbitos em 2008. No Brasil elas constituem o problema de saúde de maior magnitude e correspondem a $72 \%$ das causas de mortes. A maioria das causas de óbito neste grupo é atribuída às Doenças do Aparelho Circulatório (DAC). ${ }^{1}$ Estima-se que para o ano de 2015, 20 milhões de pessoas irão morrer de doenças cardiovasculares. ${ }^{2}$

0 município de Bandeirantes (PR) apresenta um quadro de mortalidade por DAC $13 \%$ acima da média nacional. Em 2005, estas representaram $41 \%$ do total de óbitos; obtendo proporção ainda maior, para o sexo feminino, correspondendo a mais de $43 \%$ do total de óbitos. ${ }^{3}$

A Hipertensão Arterial Sistêmica (HAS) é a causa mais frequente das DAC e o seu manejo adequado, de caráter prioritário, requer ações articuladas em três eixos: "a vigilância da hipertensão, das comorbidades e de seus determinantes; a integralidade do cuidado; e a promoção da saúde". 1:52 Neste último eixo está incluída a atividade física. Segundo a Organização Pan-Americana de Saúde, o risco de ser acometida por uma doença cardiovascular aumenta 1,5 vezes nas pessoas que não praticam atividade física mínima recomendada. ${ }^{4}$

A HAS e a inatividade física são responsáveis por $19 \%$ das mortes no mundo. ${ }^{1}$ Inquéritos populacionais nas cidades brasileiras nos últimos 20 anos estimou a prevalência da hipertensão arterial acima de $30 \%{ }^{5}$ e da atividade física no lazer na população brasileira manteve-se baixa e estável entre 2006 e 2010 (14,8\% e 14,9\%, respectivamente). ${ }^{1}$

Em um município da região metropolitana de Curitiba, no estado do Paraná, autores estimaram maior adesão à prática de atividade física por parte de usuários adultos cadastrados no programa de HAS, em cerca de $50 \% .^{6}$

A Sociedade Brasileira de Cardiologia indica para prevenção e tratamento da HAS, a prática de atividade física com duração de 30 minutos, cinco vezes por semana, de forma contínua e acumulada. ${ }^{5}$ No entanto, as mudanças de hábitos não saudáveis incorporados no cotidiano das pessoas não são fáceis de serem realizadas, o que exige do profissional de saúde a persistência na orientação em saúde para oferecer suporte e motivação para práticas saudáveis. ${ }^{7}$ As evidências científicas ressaltando os benefícios de tais práticas, subsidiam o profissional neste desafio.

Neste sentido, elaborou-se a seguinte questão norteadora: as mulheres com HAS que participam de grupos de caminhada apresentam menores médias no Escore de Risco Cardiovascular de Framinghan (ERCF) do que mulheres com HAS que não participam de grupos de caminhada? 


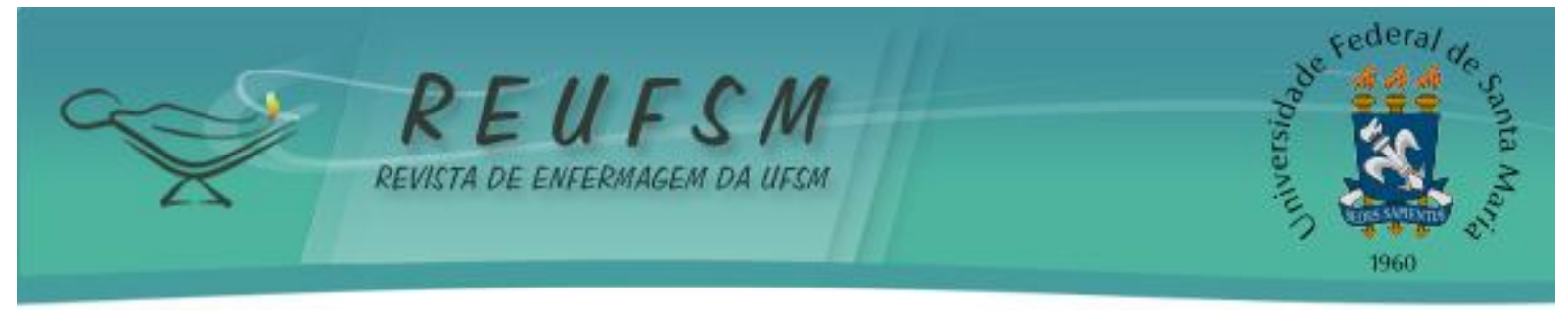

Frente ao exposto, o objetivo do presente estudo foi comparar a diferença de médias do ERCF em mulheres com HAS que participavam do grupo de caminhada com as que não participavam.

\section{MÉTODO}

Trata-se de um estudo transversal realizado em uma Unidade Básica de Saúde (UBS) com Estratégia Saúde da Família (ESF), no município de Bandeirantes, localizado na região norte do Paraná.

A população foi representada por mulheres com HAS residentes na área de abrangência da UBS. No âmbito da atenção primária à saúde, a equipe de Saúde de Família oferta a estas mulheres, consultas médicas, consultas de enfermagem, visitas domiciliares, dispensação de medicamentos para o tratamento da hipertensão arterial e grupo de caminhada. Neste grupo, as mulheres são avaliadas previamente pelo médico da UBS quanto à sua aptidão para prática da atividade física e após a inserção no grupo, participam de caminhadas três vezes por semana com supervisão da equipe de enfermagem e um professor de educação física. Participavam regularmente do grupo, 25 mulheres.

Para composição da amostra, foram convidadas as mulheres do grupo de caminhada, que preencheram os seguintes critérios de inclusão: ser mulher, ter idade acima de 30 anos, ter recebido o diagnóstico de HAS (CID I-10), estar cadastrada no SisHiperdia, participar do grupo de caminhada há mais de dois meses de forma regular, três vezes na semana e que compareceram regularmente à UBS para adquirir a medicação para tratamento da HAS no último ano.

Foram selecionadas 20 mulheres, que constituíram o Grupo 1, denominado de Grupo Caminha. Para compor o Grupo 2, foi determinado tamanho amostral na proporção 1:1 comparado ao Grupo 1. Os critérios de inclusão para o Grupo 2, denominado de Grupo Não Caminha, foram: ser mulher, ter idade acima de 30 anos, ter recebido o diagnóstico de HAS (CID I-10), estar cadastrada no Sis-Hiperdia, não participar do grupo de caminhada, não praticar atividade física no lazer e que compareceram regularmente à UBS para adquirir a medicação para tratamento da HAS no último ano. Para composição do Grupo 2Não Caminha, foram selecionadas as primeiras 20 mulheres que compareceram na UBS no mês de maio de 2008, preencheram os critérios de inclusão e consentiram participar voluntariamente da pesquisa. 0 tamanho da amostra deste estudo foi de 40 mulheres, 20 alocadas no Grupo 1-Caminha e 20 para o Grupo 2-Não Caminha.

A coleta de dados foi realizada no consultório da UBS no período de junho a agosto de 2008. Os instrumentos utilizados foram formulário do perfil sociodemográfico, clínico e laboratorial; e o ERCF.

O formulário foi composto por variáveis de sociodemográficas que incluíram dados de identificação, antecedentes pessoais específicos para riscos cardiovasculares modificáveis como: HAS, diabetes mellitus, hipercolesterolemia, tabagismo e presença de obesidade e os não-modificáveis como: idade, sexo e antecedentes familiares; as variáveis clínicas foram: peso, altura, circunferência abdominal, aferição da pressão arterial e cálculo do Índice de Massa Corpórea (IMC) de acordo com o índice de Queletec; e as variáveis laboratoriais foram: glicemia capilar, colesterol total, LDL-c, HDL-c e triglicérides.

0 ERCF foi computado como a probabilidade de desenvolvimento de um evento cardiovascular (ECV) por 10 anos, para o sexo feminino, utilizando-se a idade, colesterol total, HDL-c, tabagismo, Pressão Arterial Sistólica (PAS), Pressão Arterial Diastólica (PAD) e diabetes mellitus. Cada variável apresenta um escore que após somadas, são correlacionados ao percentual de risco para ECV nos próximos 10 anos. A classificação do risco global para o 


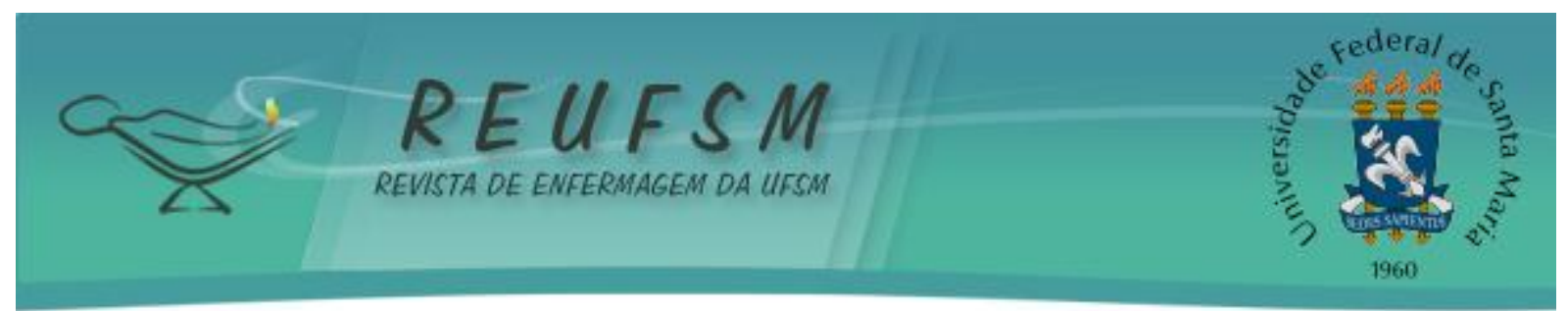

ERCF é categorizado em risco baixo $(<10 \%)$, risco moderado $(10 \%$ a $20 \%)$ ou risco alto $(>20 \%){ }^{8-9}$

As variáveis sociodemográficas foram autodeclaradas pelos participantes. Para obtenção das variáveis laboratoriais, os exames foram solicitados pelo médico da UBS e a coleta do material para exame foi realizada pelos pesquisadores em sala apropriada na UBS e armazenada em caixas térmica com controle de temperatura e transportada ao laboratório conveniado ao SUS.

A glicemia foi mensurada pelo teste de glicemia capilar com glicosimêtro da marca Optium Xceed ${ }^{\circledR}$ Abbott Diabetes Care, lote 53832 . A pressão arterial foi mensurada pelo método auscultatório com aparelho esfigmomanômetro do tipo aneroide devidamente calibrado, conforme procedimento recomendado pela VI Diretrizes de Hipertensão Arterial. ${ }^{5} \mathrm{O}$ peso e altura foram mensurados em balança antropométrica da marca Filizola ${ }^{\circledR}$. A Circunferência Abdominal (CA) foi medida utilizando-se fita métrica com escala milimétrica, colocada entre a crista ilíaca superior e a décima segunda costela passando-a na linha da cicatriz umbilical.

Foram consideradas hipertensas, mulheres que referiram ter recebido diagnóstico médico de HAS ou aquelas que estivessem fazendo uso de medicação anti-hipertensiva. Aquelas que referiram tratamento com insulina e/ou hipoglicemiante oral, foram classificados como portadoras de diabetes mellitus. Foram consideradas fumantes mulheres que fumam, independente da quantidade de cigarros.

As mulheres que apresentaram resultados alterados, tais como glicemia, perfil lipídico e/ou pressão arterial, foram orientadas e encaminhadas a consulta médica para reavaliar o tratamento, preservando a saúde da mesma e respeitando o direito do sujeito de saber sobre sua condição de saúde.

O presente estudo foi submetido ao parecer da Comissão de Ética em Pesquisa do Curso de Enfermagem da UENP/CLM, e aprovado sob o protocolo $n^{\circ} 45 / 2008$, em 27 de junho de 2008. Este estudo foi autorizado pela Secretaria de Saúde do Município e conduzido de acordo com os padrões éticos exigidos, pautado na beneficência, não maleficência, autonomia e respeito. Entregou-se juntamente ao formulário um Termo de Consentimento Livre e Esclarecido (TCLE), o qual garantiu o anonimato dos participantes, conforme resolução $n^{\circ} 196 / M S / C N S$, de 10 de outubro de $1996,{ }^{10}$ que estabelece as diretrizes e normas regulamentadoras de pesquisas envolvendo seres humanos. Foram esclarecidos ainda os objetivos do trabalho e a garantia de que não receberão nenhum ônus nem bônus ao participarem da mesma. Todos concordaram e assinaram o TCLE.

Os dados foram digitados em planilha do programa Microsoft Excel ${ }^{\circledR}$ e exportados para o software SPSS versão 13.0. Os dados foram analisados pela estatística inferencial, utilizando-se testes Qui-quadrado e T-Student para amostras independentes. Adotou-se nível de significância de 5\%.

\section{RESULTADOS}

Participaram do estudo 40 mulheres, 20 pertencentes ao Grupo 1-Caminha e 20 ao Grupo 2-Não Caminha. Apresenta-se na Tabela 1, a caracterização sociodemográfica e clínica. 


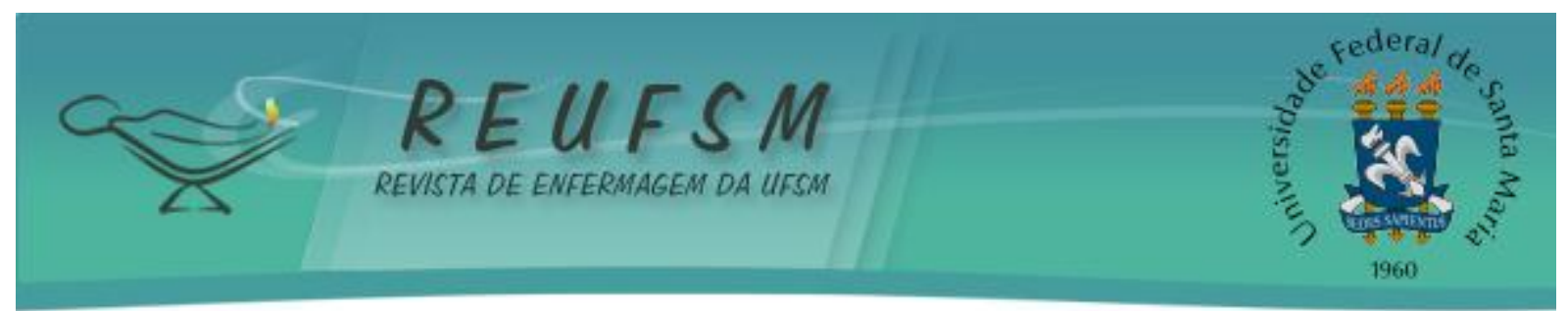

TABELA 1 - Distribuição dos sujeitos da pesquisa, de acordo com as características sociodemográficas e clínicas. Bandeirantes (PR), 2008

\begin{tabular}{|c|c|c|c|c|c|}
\hline \multirow{2}{*}{$\begin{array}{c}\text { Variáveis sociodemográficas e } \\
\text { clínicas }\end{array}$} & \multicolumn{2}{|l|}{$\begin{array}{l}\text { Grupo } 1 \\
\text { Caminha }\end{array}$} & \multicolumn{2}{|c|}{$\begin{array}{c}\text { Grupo } 2 \\
\text { Não Caminha }\end{array}$} & \multirow[t]{2}{*}{$\mathrm{p}$ value } \\
\hline & Média(DP) & $\%$ & Média(DP) & $\%$ & \\
\hline Idade & $61,00( \pm 10,40)$ & - & $60,30( \pm 8,79)$ & - & 0,819 \\
\hline Raça & & & & & 0,311 \\
\hline Negra & & 75 & & 60 & \\
\hline Branca & & 25 & & 40 & \\
\hline Escolaridade & & & & & 0,352 \\
\hline Não sabe ler ou escrever & & 20 & & 05 & \\
\hline Ensino fundamental & & 70 & & 85 & \\
\hline Ensino médio & & 10 & & 10 & \\
\hline Ensino superior & & 00 & & 00 & \\
\hline Tabagismo & & 00 & & 20 & $0,035^{*}$ \\
\hline \multicolumn{6}{|l|}{ Antecedentes pessoais } \\
\hline Dislipidemias & & 20 & & 10 & 0,378 \\
\hline Diabetes mellitus & & 20 & & 35 & 0,288 \\
\hline Infarto do Miocárdio & & 00 & & 00 & $\mathrm{n} / \mathrm{c}$ \\
\hline Doença Vascular Periférica & & 15 & & 20 & 0,677 \\
\hline Acidade Vascular Encefálico & & 10 & & 05 & 0,548 \\
\hline \multicolumn{6}{|l|}{ Antedecentes familiares } \\
\hline Hipertensão arterial & & 75 & & 80 & 0,705 \\
\hline Dislipidemias & & 20 & & 35 & 0,288 \\
\hline Diabetes mellitus & & 30 & & 60 & 0,057 \\
\hline Infarto do Miocárdio & & 20 & & 50 & $0,047^{*}$ \\
\hline Acidade Vascular Encefálico & & 45 & & 35 & 0,519 \\
\hline
\end{tabular}

Legenda: * estatisticamente significativo. Variáveis categóricas: utilizado teste Qui-quadrado. Variáveis numéricas: utilizado teste T-Student para amostras independentes. DP = Desvio padrão.

As participantes apresentaram idade média de aproximadamente 61 anos, variando entre 43 e 77 anos, sendo 67,5\% que se autodenominaram da raça branca, 17,5\% da raça negra e $15 \%$ da raça parda. Prevaleceu baixo nível de escolaridade e socioeconômico. As doenças mais prevalentes referidas pelas mulheres do grupo foram dislipidemias e diabetes mellitus, e no Grupo 2-Não Caminha, diabetes mellitus e doença vascular periférica. A HAS foi a morbidade mais prevalente nos familiares das mulheres de ambos grupos.

O perfil clínico das mulheres com HAS diferiu nas variáveis tabagismo e histórico familiar de Infarto do Miocárdio, com parentesco de $1^{\circ}$ grau. Ambos os fatores de risco foram mais prevalentes no grupo de mulheres que não participavam do grupo de caminhada. No que se refere às variáveis laboratoriais, são expressas na Tabela 2. 


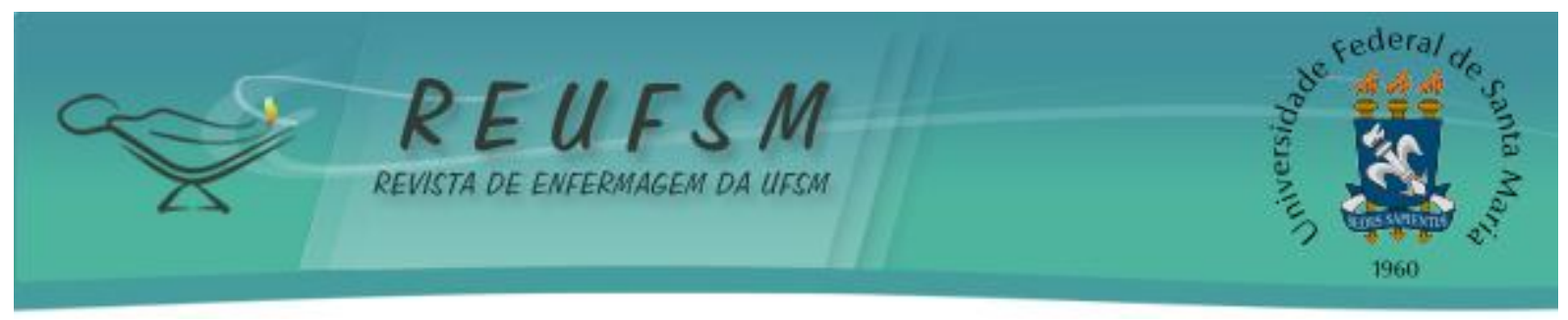

TABELA 2 - Distribuição dos sujeitos da pesquisa, de acordo com valores médios, dos resultados clínico-laboratoriais. Bandeirantes (PR), 2008.

\begin{tabular}{lccccc}
\hline \multicolumn{1}{c}{ Variáveis laboratoriais } & $\begin{array}{c}\text { Grupo } 1 \\
\text { Caminha }\end{array}$ & $\begin{array}{c}\text { Grupo } 2 \\
\text { Não Caminha }\end{array}$ & $\begin{array}{c}\text { Diferença } \\
\text { entre as } \\
\text { médias }(I C\end{array}$ & p value \\
\cline { 2 - 3 } & Média(DP) & Média(DP) & & \\
\hline Índice de Massa Corpórea $\left(\mathrm{Kg} / \mathrm{m}^{2}\right)$ & $31,04( \pm 3,62)$ & $28,70( \pm 5,95)$ & $-0,81$ a 5,49 & 0,141 \\
Circunferência abdominal $(\mathrm{cm})$ & $107,05( \pm 10,57)$ & $102,30( \pm 10,68)$ & $-2,05$ a 11,55 & 0,166 \\
Glicemia capilar $(\mathrm{mg} / \mathrm{dL})$ & $149,05( \pm 70,52)$ & $140,75( \pm 42,86)$ & $-29,06$ a 45,66 & 0,655 \\
Colesterol total $(\mathrm{mg} / \mathrm{dL})$ & $205,25( \pm 29,78)$ & $222,25( \pm 33,18)$ & $-37,18$ a 3,18 & 0,096 \\
HDL-c $(\mathrm{mg} / \mathrm{dL})$ & $54,45( \pm 11,44)$ & $50,45( \pm 9,33)$ & $-2,69$ a 10,68 & 0,233 \\
Triglicérides $(\mathrm{mg} / \mathrm{dL})$ & $135,40( \pm 51,18)$ & $160,70( \pm 70,16)$ & $-64,61$ a 14,01 & 0,200 \\
Pressão Arterial Sistólica $(\mathrm{mmHg})$ & $130,50( \pm 14,69)$ & $150,50( \pm 16,70)$ & $-30,06$ a $-9,94$ & $0,000^{*}$ \\
Pressão Arterial Diastólica $(\mathrm{mmHg})$ & $85,50( \pm 8,26)$ & $93,50( \pm 14,61)$ & $-15,60$ a $-0,40$ & $0,040^{*}$
\end{tabular}

Legenda: * estatisticamente significativo ao nível de significância de 95\%. Variáveis categóricas: utilizado teste Qui-quadrado. Variáveis numéricas: utilizado teste T-Student para amostras independentes. $\mathrm{DP}=$ Desvio padrão.

O Grupo 1-Caminha apresentou média de IMC de $31,04 \mathrm{Kg} / \mathrm{m}^{2}$, enquadrando-se em obesidade grau 1, sendo a CA média de 107,05 cm. Já o Grupo 2-Não Caminha obteve média de IMC de $28,7 \mathrm{Kg} / \mathrm{m}^{2}$, sendo classificado em sobrepeso, com CA média de $102,3 \mathrm{~cm}$.

As variáveis glicemia capilar, colesterol total, HDL-c e triglicérides não apresentaram diferença estatística entre os grupos. A média da glicemia em jejum estava acima do limite considerado normal pela Sociedade Brasileira de Diabetes. ${ }^{11}$

Em relação a pressão arterial, houve diferença estatística entre as médias dos níveis de PAS e PAD dos grupos, sendo o Grupo 1-Caminha com valores menores que o Grupo 2-Não Caminha.

Assim salienta-se na Tabela 3, a distribuição dos sujeitos do estudo, de acordo com o controle da pressão arterial (PA $\leq 140 / 90 \mathrm{mmHg}$ ), conforme proposto pela $\mathrm{VI}$ Diretrizes de Hipertensão Arterial.

Tabela 3 - Proporção de mulheres com Hipertensão Arterial Sistêmica com controle da enfermidade (PA $\leq 140 / 90 \mathrm{mmHg}$ ), conforme proposto pela VI Diretriz de Hipertensão Arterial. Bandeirantes (PR), 2008

\begin{tabular}{|c|c|c|c|c|c|}
\hline \multirow{2}{*}{$\mathrm{PA} \leq 140 / 90 \mathrm{mmHg}$} & \multicolumn{2}{|c|}{$\begin{array}{l}\text { Grupo } 1 \\
\text { Caminha }\end{array}$} & \multicolumn{2}{|c|}{$\begin{array}{c}\text { Grupo } 2 \\
\text { Não Caminha }\end{array}$} & \multirow{2}{*}{$\mathrm{p}$ value } \\
\hline & (n) & $(\%)$ & (n) & (\%) & \\
\hline Sim & 06 & 30 & 01 & 05 & \\
\hline Não & 14 & 70 & 19 & 95 & $0,037^{*}$ \\
\hline
\end{tabular}

Legenda: ${ }^{*}$ estatisticamente significativo ao nível de significância de 95\%.

0 percentual de mulheres com controle pressórico foi superior no Grupo 1Caminha. Contudo, 95\% dos indivíduos pertencentes ao Grupo 2-Não Caminha e 70\% do Grupo 1-Caminha, não apresentaram pressão arterial controlada ( $\leq 140 / 90 \mathrm{mmHg}$ ). 


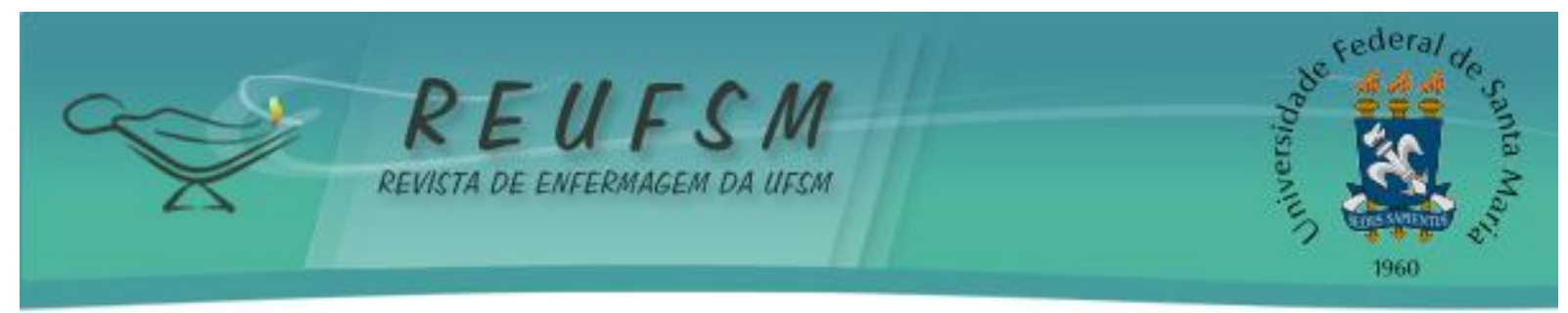

No que se refere ao risco de desenvolvimento de eventos cardiovasculares, calculouse o ERCF, e a comparação da média geral da pontuação obtida é apresentada na Tabela 4.

TABELA 4 - Distribuição do escore médio obtido e percentual de risco atribuído aos grupos do estudo, baseado no Escore de Risco Cardiovascular de Framinghan (ERCF). Bandeirantes (PR), 2008.

\begin{tabular}{|c|c|c|c|}
\hline Escore absoluto obtido no ERCF & Média & $\begin{array}{c}\text { Diferença entre as } \\
\text { médias (IC } 95 \% \text { ) }\end{array}$ & p value \\
\hline Grupo 1 - Caminha & 8,85 & \multirow[b]{2}{*}{$-5,994$ a $-0,606$} & \multirow[b]{2}{*}{$0,018^{*}$} \\
\hline Grupo 2 - Não Caminha & 12,15 & & \\
\hline \% de Risco segundo Framinghan & (\%) & $\begin{array}{c}\text { Diferença entre as } \\
\text { médias (IC } 95 \% \text { ) }\end{array}$ & p value \\
\hline Grupo 1 - Caminha & $9,30 \%$ & \multirow[b]{2}{*}{$-10,524$ a $-1,676$} & \multirow[b]{2}{*}{$0,008^{*}$} \\
\hline Grupo 2 - Não Caminha & $15,40 \%$ & & \\
\hline
\end{tabular}

Legenda: * estatisticamente significativo ao nível de significância de 95\%.

Evidencia-se que houve diferença estatisticamente significativa das médias das pontuações obtidas pelos grupos. O Grupo 1-Caminha apresentou pontuação média por meio do ERCF de 8,85, variando entre 3 a 17 pontos, classificando-se como baixo risco para desenvolvimento de doenças cardiovasculares. Já o Grupo 2-Não Caminha revelou pontuação média de 12,15 , com amplitude 2 a 20 pontos, caracterizando como médio risco.

\section{DISCUSSÃO}

As mulheres participantes desta pesquisa encontravam-se na faixa etária de risco para doenças cardiovasculares, pois, para mulheres, idade acima de 55 anos representa um indicador intermediário de risco. ${ }^{12}$

No que se refere às medidas clínicas, o cálculo do IMC é uma medida importante que vem sendo comumente utilizada para classificar adultos com sobrepeso ou obesos, pois indivíduos que apresentam IMC entre 25 e $29,9 \mathrm{Kg} / \mathrm{m}^{2}$ são considerados pré-obesos, condição que representa fator adicional para risco cardiovascular ${ }^{12}$, pois o aumento do tecido adiposo central está fortemente relacionado com as doenças cardiovasculares ateroscleróticas. ${ }^{13}$

Pessoas com sobrepeso ou obesas apresentam mais chances de portar problemas de saúde do que aquelas classificadas como eutróficas. Entre as pessoas cadastradas em uma UBS com ESF de Nova Andradina-MS, a presença da HAS sozinha ou associada a outras doenças mostrou 3,3 vezes mais chances de risco em indivíduos obesos. ${ }^{14}$

0 valor médio de IMC obtido pelo Grupo 2-Não Caminha assemelhou-se ao estudo realizado com portadores de HAS no município de Brusque (SC), onde foi observada média entre as mulheres de $28,1 \mathrm{Kg} / \mathrm{m}^{2} .^{15}$

Outro índice antropométrico avaliado foi a CA. Nota-se que os dois grupos apresentaram medida da CA acima do recomendado, visto que para mulheres a medida ideal é menor de $88 \mathrm{~cm} .{ }^{16} \mathrm{Em}$ um estudo com funcionárias públicas de uma universidade do Estado do Rio de Janeiro, mostrou que mulheres com valores de CA elevados apresentaram mais chances de terem HAS e a razão de prevalência seguiu o mesmo padrão da associação da obesidade com HAS, 2,45 e 2,32, respectivamente. ${ }^{17}$

Em relação ao nível de triglicerídeos, colesterol total e HDL-c o Grupo 1-Caminha apresentou resultados melhores que o Grupo 2-Não Caminha. Indivíduos ativos apresentam níveis 


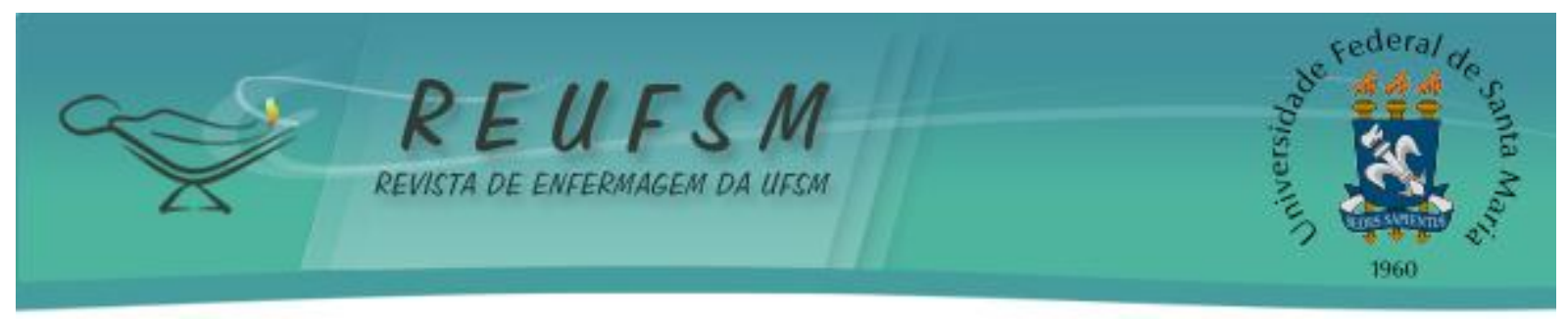

mais baixos de colesterol, triglicerídeos e LDL-c e concentrações mais elevadas de HDL-c, em relação aos inativos, ${ }^{18}$ o que explica os melhores resultados verificados no Grupo 1-Caminha.

As mulheres do Grupo 2-Não Caminha, apresentaram um incremento do risco ao desenvolvimento de doenças cardiovasculares, quando comparado ao Grupo 1-Caminha, pois o colesterol é um fator de risco decisivo para a aterogênese. Dados do Ambulatório de Cardiologia e Hipertensão Arterial do Centro de Saúde Escola da Faculdade de Medicina de Ribeirão Preto da Universidade de São Paulo, envolvendo 382 pessoas, evidenciou que entre os pacientes classificados como alto risco para ECV segundo o ERCF, houve maior percentual de mulheres com níveis de triglicérides acima do normal quando comparado ao percentual de homens. ${ }^{19}$

Apesar do Grupo 1-Caminha ter apresentado fatores agravantes como obesidade, a caminhada mostrou ser eficaz na redução de outros fatores de risco como HAS, colesterol total e HDL-c, que determinou menor pontuação e, consequentemente, menor risco para desenvolvimento de ECV que o Grupo 2-Não Caminha.

Outro indicador clínico utilizado foi a pressão arterial, considerada um dos mais significativos fatores de risco para as principais doenças cardiovasculares ${ }^{12}$, pois possui uma forte relação com $80 \%$ dos casos de Acidente Vascular Cerebral e $60 \%$ dos casos de doença isquêmica do coração. ${ }^{20}$

Observou-se que 30\% do Grupo 1-Caminha apresentavam níveis pressóricos controlados, superando assim os resultados encontrados em outro estudo brasileiro, o qual revelou que $89,6 \%$ de indivíduos com HAS não tinham pressão arterial controlada. ${ }^{2} \mathrm{Em}$ Fortaleza-CE, uma pesquisa ${ }^{21}$ envolvendo 246 pessoas com HAS cadastradas em seis UBS com ESF revelou que $28 \%$ delas apresentavam pressão arterial em níveis controlados, proporção próxima da verificada no Grupo 1-Caminha.

Ainda, em relação aos níveis pressóricos, o Grupo 1-Caminha apresentou valores médios de Pressão Arterial Sistólica (PAS) de 130,5 mmHg e Pressão Arterial Diastólica (PAD) de $85,5 \mathrm{mmHg}$, se enquadrando dentro das metas preconizados pela VI Diretrizes de Hipertensão arterial. O Grupo 2-Não Caminha, manifestou valores médios mais elevados, com PAS de 150,5 mmHg e PAD 93,5 mmHg, acima da meta proposta pela VI Diretrizes de Hipertensão arterial de $140 / 90 \mathrm{mmHg} .{ }^{5}$

Um estudo quase experimental desenvolvido pela Facultad de Enfermería y Obstetricia de la Universidad Autónoma del Estado de México, em 2009, demonstrou efeito favorável de um programa de exercício físico para redução da pressão arterial de pessoas com HAS. O estudo envolveu 110 pessoas que após a participação no referido programa, obtiveram redução média de $8,28 \mathrm{mmHg}$ na PAS e 4,72 na PAD. ${ }^{22^{3}}$

No entanto, apesar dos estudos terem destacados os benefícios da atividade física, a frequência de adultos que praticam atividade física oscila entre $11,3 \%$ na população da cidade do Rio Branco à 22,4\% no Distrito Federal. Em Curitiba, capital do estado onde se realizou o estudo em tela, $13,8 \%$ referiram praticar atividade física no lazer, sendo o maior percentual referido por homens. ${ }^{23}$ Estudos tem recomendado enfaticamente a adoção da prática de atividade física como uma das ações para adoção de um estilo de vida saudável. 14,17,21-22 $^{2}$

\section{CONCLUSÃO}

A participação no grupo de caminhada revelou ser um fator diferencial no controle da maioria dos fatores de risco, evidenciando assim que a caminhada é um importante fator de proteção para as doenças cardiovasculares, uma vez que, mesmo que integrantes do grupo de caminhada apresentaram alguns fatores de risco mais prevalentes como a obesidade, em relação ao grupo não praticante, essas mulheres atingiram menores pontuações por meio do ERCF.

Portanto, a caminhada representa uma estratégia para prevenção de doenças cardiovasculares, podendo ser utilizada como tratamento coadjuvante dos principais 


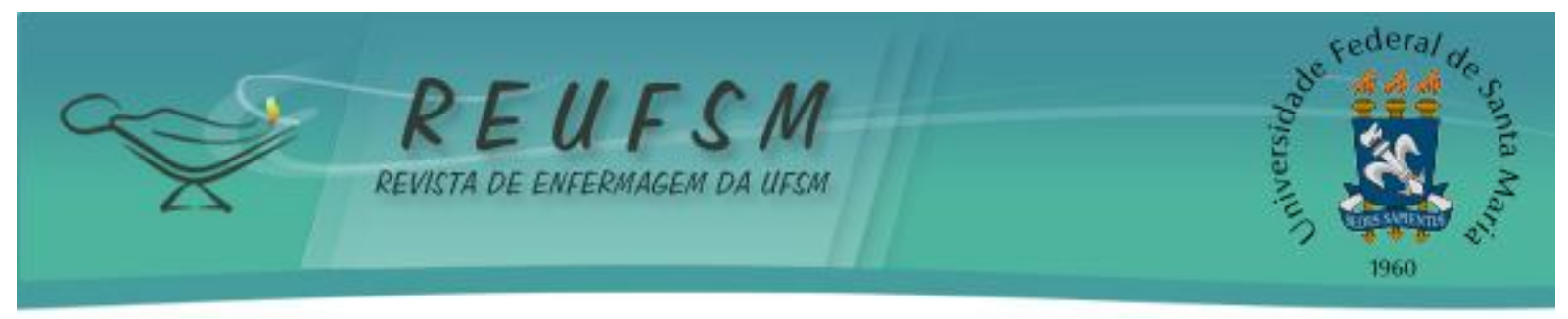

fatores de risco modificáveis. Por se tratar de uma ação de baixo custo e de fácil acesso, os grupos de caminhada tem potencialidade para serem difundidos nas UBS com ESF, como medida para prevenção secundária às mulheres com HAS.

O ERCF configurou-se como uma metodologia factível na atenção primária à saúde, com foco na prevenção secundária, pois as evidências deste estudo servem de informação para motivação do grupo de caminhada a manterem a adesão nesta estratégia de prevenção, tão importante para redução de mortes e danos advindos de causas cardiovasculares.

Considerando o grupo não praticante, se faz necessário um trabalho intensivo de incentivo por parte das unidades de saúde e até mesmo com o auxílio do grupo praticante para estimular a participação nas caminhadas, através de reuniões com a comunidade para uma conscientização da importância da caminhada na prevenção destes agravos. Este estudo apresenta limitações de generalização dos resultados inerentes ao tamanho da amostra. $O$ delineamento deste estudo não possibilita a avaliação do efeito da participação no grupo de caminhada sobre o ERCF, por não se conhecer as variáveis antes do ingresso das mulheres neste grupo.

\section{REFERÊNCIAS}

1. Brasil. Plano de enfrentamento das doenças crônicas não transmissíveis (DCNT) no Brasil 2011/2012. Brasília: Ministério da Saúde, 2011. 148 p.

2. World Health Organization. Cardiovascular diseases [internet]. [acesso em 2008 Jun 12]. Disponível em: http://www.who.int/cardiovascular_diseases/en

3. Brasil. Ministério da Saúde. Sistema de Informações sobre Mortalidade - SIM/DATA-SUS [internet]. [acesso em 2008 Jun 19]. Brasil. Disponível em:

http: / / tabnet.datasus.gov.br/cgi/deftohtm.exe?sim/cnv/obtuf.def

4. Organização Pan-Americana da Saúde. Doenças crônico-degenerativas e obesidade: Estratégia mundial sobre alimentação saudável, atividade física e saúde. Brasília (Brasil): 2003. 60p.

5. Sociedade Brasileira de Cardiologia. VI Diretrizes Brasileira de Hipertensão. Brazilian Journal of Hypertension. 2010;17(1):1-64.

6. Mantovani MF, Mottin JV, Ulbrich EM, Pinotti S. Health clients profile and knowledge about arterial hypertension. Online Brazilian Journal of Nursing [internet]. 2008 [acesso em 2012 Mai 26]; 7(2). Disponível em:

http://www.objnursing.uff.br/index.php/nursing/article/view/j.1676-4285.2008.1467

7. Mantovani MF, Mottin JV, Rodrigues J. Nurse home visit with educational activities in the arterial pressure treatment. Online Brazilian Journal of Nursing [internet]. 2007 [acesso em 2012 Mar 1]; 6(1). Disponível em: http://www.objnursing.uff.br/index.php/nursing/article/view/757

8. Fernandes CE, Pinho-Neto JSL, Gebara OCE, Santos Filho RD, Pinho-Neto AM, Pereira Filho AS et al. I Diretriz Brasileira sobre Prevenção de Doenças Cardiovasculares em Mulheres Climatéricas e a Influência da Terapia de Reposição Hormonal (TRH) da Sociedade Brasileira de Cardiologia (SBC) e da Associação Brasileira do Climatério (SOBRAC). Arq Bras Cardiol. 2008; 91(1 Supl. 1):1-23.

9. Sampaio MR, Melo MBO, Wanderley MSA. Risco cardiovascular global em pacientes de USF. Rev Bras Cardiol. 2010; 23(1):47-56. 


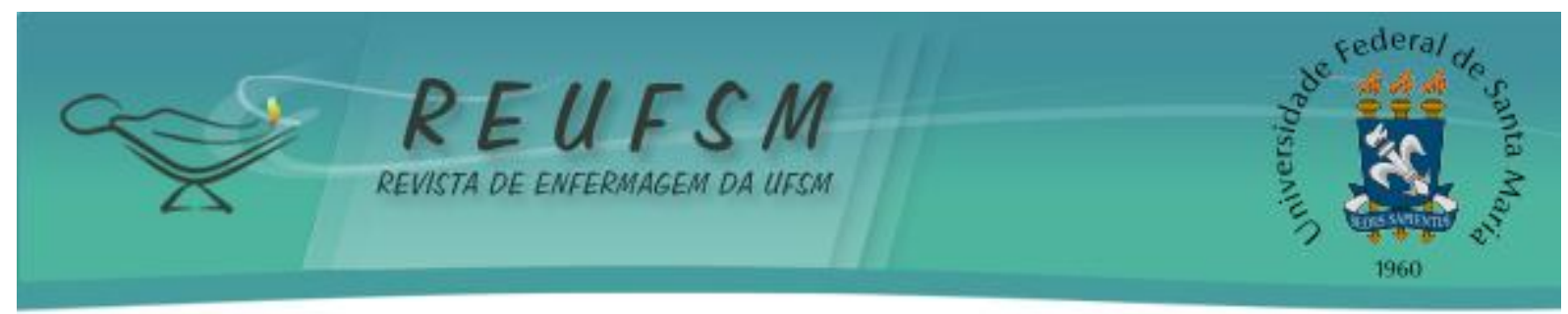

10. Conselho Nacional de Saúde, Brasil. Resolução n¹96/MS/CNS, de 10 de outubro de 1996 [internet]. [acesso em 2008 Ago 15]. Disponível em:

http:/ / conselho.saude.gov.br/docs/Resolucoes/Reso196.doc

11. Sociedade Brasileira de Diabetes. Algoritmo para tratamento do diabetes tipo 2. Atualização 2009. Posicionamento oficial $n^{\circ} 2.2009 .26$ p.

12. Organização Pan-Americana da Saúde. Linhas de cuidado: hipertensão arterial e diabetes. / Organização Pan-Americana da Saúde. Brasília: Organização Pan-Americana da Saúde, 2010. 232 p.: il.

13. Guimarães DED, Sardinha FLC, Mizurini DM, Carmo MGT. Adipocitocinas: uma nova visão do tecido adiposo. Rev Nutr. 2007; 20(5):549-59.

14. Amer NM, Marcon SS, Santana RG. Body mass índex and hypertension in adults subjects in Brazil's Midwest. Arq Bras Cardiol. 2011; 96(1):47-53.

15. Rosini N, Machado MJ, Xavier HT. Estudo de prevalência e multiplicidade de fatores de risco cardiovascular em hipertensos do município de Brusque, SC. Arq Bras Cardiol. 2006; 86(3):219-22.

16. Associação Brasileira para o Estudo da Obesidade e da Síndrome Metabólica. Diretrizes brasileiras de obesidade 2009/2010 / ABESO. $3^{\text {a }}$ ed. - Itapevi, SP: AC Farmacêutica, 2009. 85p.

17. Hasselmann MH, Faerstein E, Werneck GL, Chór D, Lopes CS. Associação entre circunferência abdominal e hipertensão arterial em mulheres: Estudo Pró-Saúde. Cad Saúde Pública. 2008; 24(5):1187-91.

18. Zanella AM, Souza DRS, Godoy MF. Influência do exercício físico no perfil lipídico e estresse oxidativo. Arquivos de Ciência da Saúde. 2007; 14(2):107-12.

19. Cesarino EJ, Vituzzo ALG, Sampaio JMC, Ferreira DAS, Pires HAF, Souza L. Assessment of cardiovascular risk of patients with arterial hypertension of a public health unit.

Einstein. 2012; 10(1):33-8.

20. Medeiros JEO, Guimarães CF. Sementes do SUS. Sapucaia do Sul: IBSaúde, 2007. 269 p.

21. Guedes MVC, Araujo TL, Lopes MVO, Silva LF, Freitas MC, Almeida PC. Barreiras ao tratamento da hipertensão arterial. Rev Bras Enferm. 2011; 64(6):1038-42.

22. Reza CG, Sánchez PBM, Pilar MM, Castro MEM. Ejercicio físico con ritmo: intervención de enfermería para el control de la hipertensión arterial en un município de el Estado de Mexico. Esc Anna Nery. 2011; 15(4):717-22.

23. Brasil. Ministério da Saúde. VIGITEL Brasil 2010: vigilância de fatores de risco e proteção para doenças crônicas por inquérito telefônico. Brasília: Ministério da Saúde, 2011. 152 p.: Il.

Data de recebimento: $27 / 05 / 2012$

Data de aceite: 10/07/2012

Contato com autor responsável: Luciano Faustino da Silva.

Endereço: Rua Moisés Lupion, 242. Arapoti-PR.

CEP: 84990-000

E-mail: lucianojbt@hotmail.com 